Andrei B. Borisov · Eduard I. Dedkov

Bruce M. Carlson

\title{
Abortive myogenesis in denervated skeletal muscle: differentiative properties of satellite cells, their migration, and block of terminal differentiation
}

Accepted: 17 September 2004 / Published online: 11 March 2005

(C) Springer-Verlag 2005

\begin{abstract}
Little is known about the biological properties of myogenic satellite cells during postdenervation muscle atrophy. The present study investigated the differentiative capacity of satellite cells and their involvement in the compensatory regenerative process in long-term denervated rat muscle. Electron microscopy and immunocytochemical labeling of muscle tissue 1-18 months following denervation demonstrated that despite activation of satellite cells, myogenesis in denervated muscle is abortive and does not lead to the formation of normal muscle fibers. Small sizes, poor development of the contractile system in newly formed denervated myotubes, and the absence of satellite cells on the surface indicate that their differentiation typically does not progress to terminal stages. Many immature myotubes degenerate, and others survive but are embedded in a collagen lattice near their parent fibers. Interestingly, newly formed myotubes located on the surface of parent muscle fibers beneath the basal lamina typically did not contain developed myofibrils. This suggests that the contacts of daughter and parent muscle fibers block myofibrillogenesis. Assembly of sarcomeres in most cases occurs following complete spatial separation of daughter and parent muscle fibers. Another manifestation of the involvement of myogenic precursors in abortive myogenesis is the formation of clusters of underdeveloped branching myotubes surrounded by a
\end{abstract}

\footnotetext{
A. B. Borisov $(\square)$

Division of Pediatric Cardiology, Department of Pediatrics and Communicable Diseases, University of Michigan, Room 8200, MSRB III, Ann Arbor, MI 48109, USA

E-mail: aborisov@umich.edu

Tel.: + 1-734-9368663

Fax: + 1-734-6151386

E. I. Dedkov · B. M. Carlson

Department of Cell and Developmental Biology, University of Michigan, Ann Arbor, MI 48109-0616, USA

B. M. Carlson

Institute of Gerontology, University of Michigan, Ann Arbor, MI 48109-0616, USA
}

common basal lamina. We found that myoblasts can also fuse directly with differentiated muscle fibers. The presence of satellite cells near the openings in the basal lamina and in the interstitial space indicates that myogenic precursors can migrate through the basal lamina and form myotubes at a distance from parent fibers. Our data may explain why long-term denervated skeletal muscle has a poor capacity for regeneration and functional restoration.

Keywords Skeletal muscle - Denervation · Myogenesis $\cdot$ Satellite cells

\section{Introduction}

Innervation is an important trophic and regulatory factor controlling terminal differentiation, stability of the differentiated phenotype, and functional activity of skeletal muscle cells. The loss of innervation results in progressive atrophy of muscle tissue and rapid impairment of its functional capacity. Structural changes and the dynamics of the atrophic process in denervated skeletal muscle at microscopic and ultrastructural levels have been described in a number of publications (see Schmalbruch and Lewis 1994; Lu et al. 1997; Borisov and Carlson 2000; Borisov et al. 2001). More than 40 years of electron microscopic and histological studies have provided detailed descriptions of fine structural characteristics of denervated muscle. At the same time, little is known regarding the cellular mechanisms that underlie postdenervation muscle atrophy. Understanding of the molecular pathways of myogenesis and the recent development of probes specific for different stages of this process opens new horizons for causal analysis of the cellular basis and pathogenesis of postdenervation muscle atrophy. One of the key issues that are directly related to the mechanisms of this process is the elucidation of the differentiative properties and activity of myogenic satellite cells. This reserve of myoblasts 
normally provides the cellular basis for successful regeneration following even minimal injury of skeletal muscle tissue.

For these reasons, the scope of our current studies is focused on the following two questions: (1) Why don't satellite cells provide compensatory regeneration of muscle tissue during the course of postdenervation atrophy? (2) Why doesn't denervated muscle recover its structural and functional properties if reinnervation occurs later than the first couple of months following denervation? The answers to these questions will allow us to determine why the high regenerative potential of skeletal muscle tissue is not realized during the atrophic process induced by denervation. Thus, the purpose of this paper was to investigate the properties of satellite cells in long-term denervated rat skeletal muscle and their involvement in compensatory regenerative reaction during the course of acute postdenervation atrophy.

\section{Materials and methods}

\section{Muscle denervation}

The experiments were conducted on adult (4-month-old) male WI/HicksCar rats maintained at the animal facility at the Department of Biology, University of Michigan. In all experiments the animals were anesthetized with ether. The right legs of the rats were denervated by sectioning the sciatic nerve high in the thigh, suturing the proximal and distal stumps, and implanting the proximal stump into hip muscles and the distal stump into the popliteal space. This procedure allows a permanent and complete denervation of the lower leg (Carlson and Faulkner 1988). The animals were euthanized under ether anesthesia at 1, 2, 4, 7, 12, 18, or 23 months after denervation.

\section{Experimental design}

Tissue samples of the extensor digitorum longus and tibialis anterior muscles were taken from four animals for each time point. Each muscle was divided into two parts, cryoprotected in a graded sucrose series, and embedded in molds for transverse and longitudinal sectioning in cryoprotective freezing medium TBS (Triangle Biomedical Sciences, Durham, NC, USA) for immunohistochemical and histological studies. Crosssections were prepared through the equatorial area of each muscle and mounted in groups of four on histological slides. Three to five slides per each time point were studied from each animal.

\section{Electron microscopy}

Samples of muscle tissue were prefixed immediately after excision from the limb in an ice-cold solution of $2.5 \%$ glutaraldehyde and $4 \%$ formaldehyde in $0.1 \mathrm{M}$ isoosmotic phosphate buffer, $\mathrm{pH} 7.4$, for $1 \mathrm{~h}$. The tissue was then cut into several smaller pieces and fixed for an additional $4 \mathrm{~h}$ at $3^{\circ} \mathrm{C}$ in fresh aliquots of fixative. After removal of fixative, the samples were washed in three changes for $15 \mathrm{~min}$ each in $0.1 \mathrm{M}$ phosphate buffer. The tissue was postfixed with $1 \% \mathrm{OsO}_{4}$ in $0.1 \mathrm{M}$ phosphate buffer for $1.5 \mathrm{~h}$ at $3^{\circ} \mathrm{C}$, washed again in the buffer solution three times (15 min each wash), and dehydrated through a graded ethanol series to absolute ethanol. After infiltration with epoxy resin, the samples were embedded in Spurr medium (Ted Pella, Redding, CA, USA). After polymerization of the medium in blocks, ultrathin sections were prepared using a Reichert-Young Ultracut E ultramicrotome and mounted on copper/ rhodium mesh grids M150-CR (Electron Microscopy Sciences, Fort Washington, PA, USA). After staining with uranium acetate and lead citrate (Reynolds 1963),

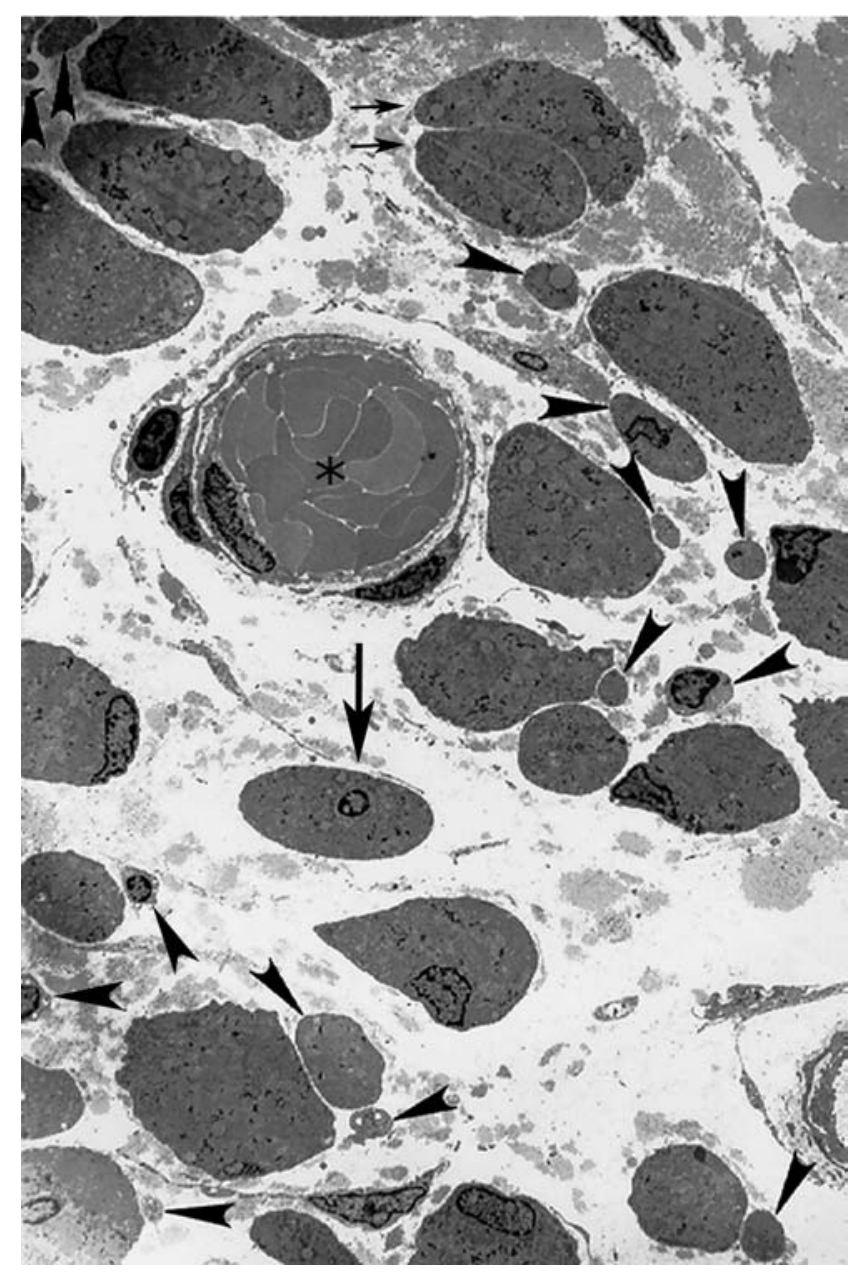

Fig. 1 Electron micrograph of a transverse section of 18-month denervated extensor digitorum longus. Arrowheads indicate small myotubes typically located at a close proximity to muscle fibers. The arrow indicates a muscle fiber with a centrally located nucleus. Two small arrows show two closely apposed muscle fibers of approximately the same size. The asterisk shows a blood vessel. $(\times 1,410)$ 
the sections were examined with a Philips CM 100 electron microscope at an accelerating voltage of $60 \mathrm{kV}$.

Immunocytochemistry and light microscopy

After the fixative was washed out, the samples were sectioned into $8-\mu \mathrm{m}$ longitudinal and cross-sections and immunostained using the technique of indirect immunofluorescence. Monoclonal antibodies to embryonic, fast, and slow isoforms of myosin and laminin were obtained from the Developmental Studies Hybridoma Bank (University of Iowa, Iowa City, IA, USA), Chemicon International (Temecula, CA, USA), and Novocastra Laboratories (Newcastle upon Tyne, UK). Fluorescein-conjugated rabbit anti-mouse IgG (Sigma, St. Louis, MO, USA) was used as a secondary antibody for detecting binding of monoclonal antibodies. TRITCconjugated goat anti-rabbit IgG (Sigma) was used as a secondary antibody for visualizing binding of polyclonal primary rabbit antibodies. The antibodies were diluted according to the recommendations of the producers. The

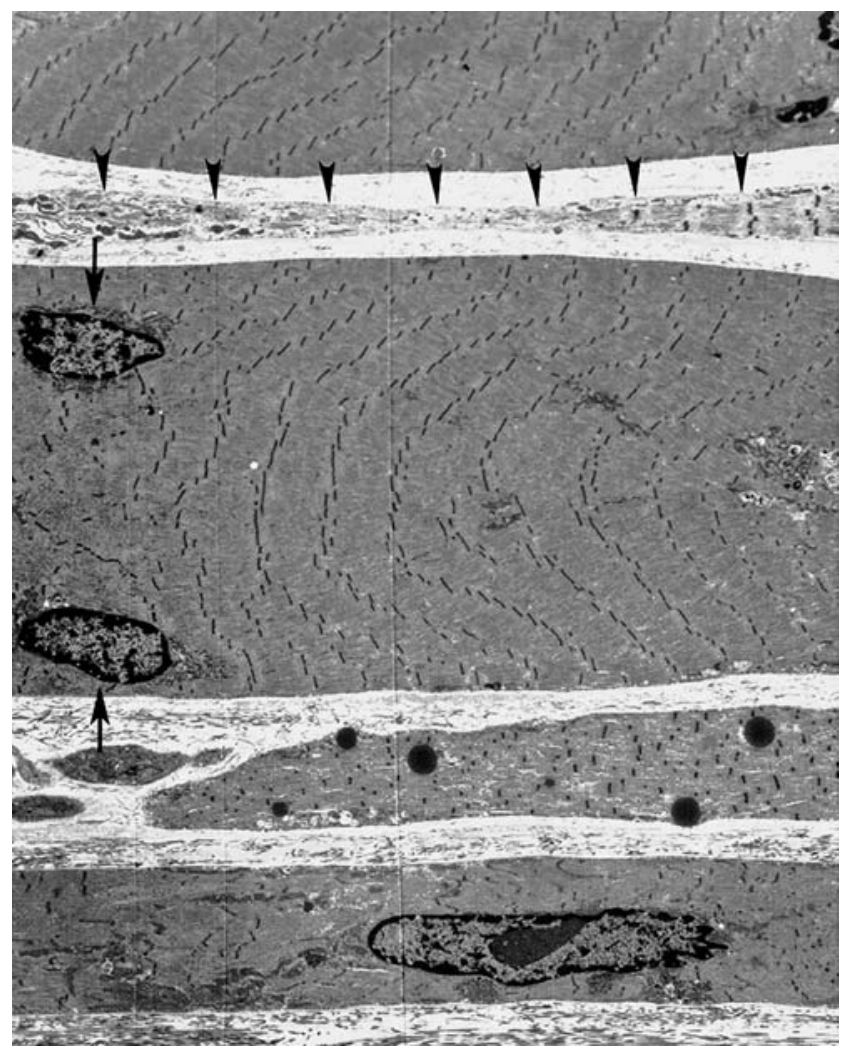

Fig. 2 Electron micrograph of a longitudinal section of 18-month denervated muscle. Arrowheads indicate a muscle fiber of small diameter with a poorly developed contractile system. The muscle fiber in the center retains a high level of integrity of the contractile system, alignment of Z-bands of myofibrils in register, and nuclei located at the periphery (short arrows), which is typical of terminally differentiated muscle cells. The muscle fiber at the bottom has a less well organized contractile system and a centrally located nucleus $(\times 2,890)$ immunofluorescent reactions were examined with a Carl Zeiss LSM 510 Meta confocal microscope and a Nikon Optiphot fluorescence microscope equipped with an
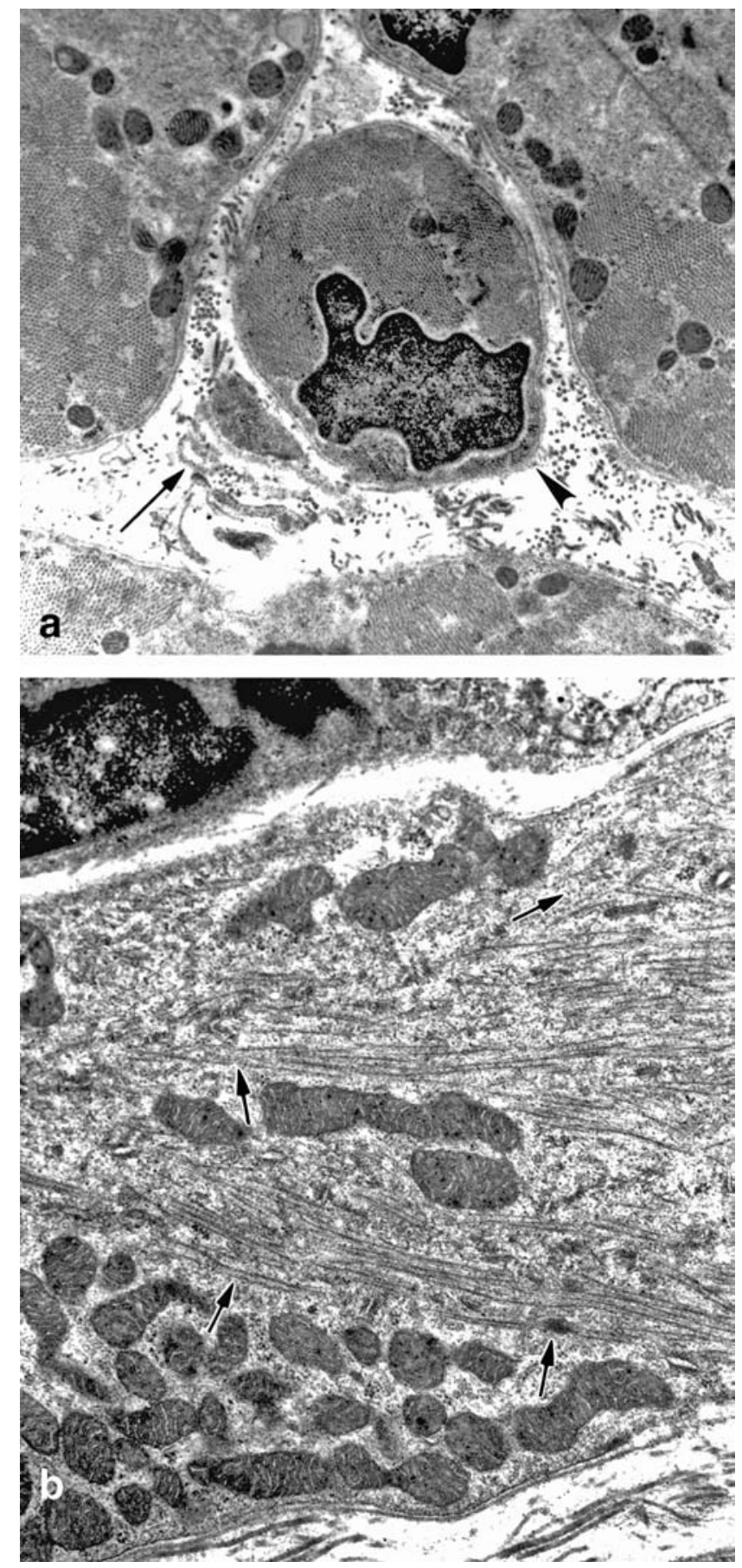

Fig. 3 Fine structure of muscle fibers of small diameter. a Electron micrograph of a transversely sectioned muscle fiber of small diameter located between three muscle fibers retaining a high level of differentiation; the arrowhead indicates the basal lamina surrounding a small fiber, and the arrow shows the empty basal lamina with a small diameter. b Electron micrograph of a longitudinally sectioned muscle fiber of small diameter shows the presence of poorly organized myofilaments (arrows) in the cytoplasm 2 months following denervation. $(\mathbf{a} \times 11,500, \mathbf{b} \times 23,000)$ 
excitation filter at 470-490 $\mathrm{nm}$ and an emission barrier filter at 510-560 nm wavelengths for fluorescein, and an excitation filter at 510-560 $\mathrm{nm}$ and an emission barrier filter at 590-615 $\mathrm{nm}$ for TRITC.

\section{Results}

Electron microscopic study of long-term denervated skeletal muscle revealed the presence of a large number of muscle fibers of very small diameter (Figs. 1, 2, 3, 4). These fibers were typically associated topographically with fibers of a much larger size (Figs. 1,2). Small and large fibers exhibited three- to 20 -fold differences in their cross-sectional areas. The topographical apposition of small and large fibers was especially evident in 7-18month denervated muscle, in which individual cells formed pairs or triplets located closely together and spatially separated from other doublets and triplets by vast deposits of collagen (Fig. 1). Small muscle fibers were frequently located so close to large fibers that they fit into sarcolemmal invaginations of the large fibers. By $10-18$ months following denervation, up to $20-25 \%$ of muscle cell population consisted of small fibers. At this late stage of denervation, some fibers in the doublets and triplets had similar sizes (Fig. 1).

It was of special interest to study the origin and the mechanism of formation of small muscle cells. The structural characteristics of small fibers indicate that they formed de novo rather than representing a severely atrophic subpopulation of terminally differentiated myocytes. These cells had smooth unfolded basal lamina

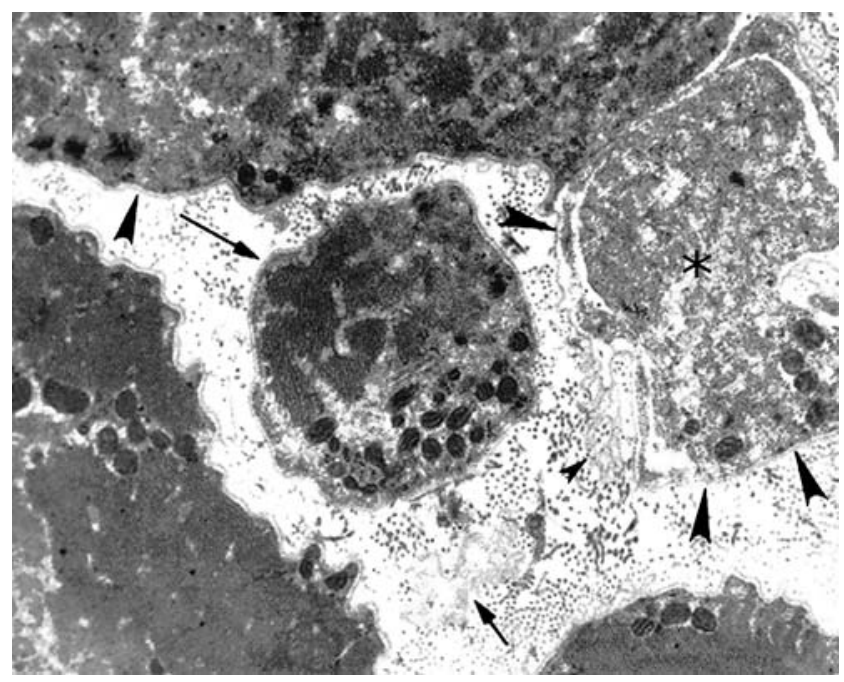

Fig. 4 Formation of a muscle fiber of small diameter (asterisk) beneath the basal lamina (arrowheads) of a parent muscle fiber. The long arrow shows another small fiber containing bundles of myofibrils and located in the endomysial space. The short arrow shows an empty basal lamina of small diameter. Note the absence of myofibrils in the nascent fiber located beneath the folded basal lamina (marked with a small arrowhead) and well-developed myofibril bundles in the fiber located outside the basal lamina. $(\times 8,500)$
(Figs. 3, 4). Unlike the small fibers, the basal laminae of severely atrophic fibers had multiple folds as a result of the decrease, or "shrinkage," of muscle cell volume during the postdenervation period, as shown in Figs. 4 and $5 \mathrm{f}$. We found that small myocytes had already started to develop in 2-month denervated muscle (Fig. 3). They were intensely immunopositive for the embryonic isotype of myosin (Figs. 5a, 6), contained myofilaments and nascent myofibrils (Fig. 3), and were spatially separated from other muscle fibers (Figs. 3, 4). Satellite cells were not seen on their surface. Figs. 1, 3, and 4 clearly show the multifold difference in sizes between the small fibers and the terminally differentiated muscle cells. Typically, there were no breaks, discontinuities, or bridges of the basal laminae in transverse and longitudinal sections of the large parent muscle fibers and the small fibers associated with them (Figs. 2, 3, 4), which indicates their clear structural separation. Empty profiles of basal laminae of small diameter were frequently found near small muscle fibers (Figs. 3a, 4, 5f). Judging by the size of these structures, we suggest that they may represent the remnants of previous generations of degenerated small fibers. This conclusion was supported by the presence in the denervated tissue of degenerating and dead small fibers (Fig. 5d-f). Interestingly, some daughter myotubes degenerated and died while still under the basal laminae of parent muscle fibers (Fig. 5d, e). It is important that in 2-4-month denervated muscle, small myotubes located beneath the basal laminae of parent muscle fibers did not contain myofibrils with more or less mature sarcomeric organization (Figs. 4, 5c-e). This may indicate that the contact of nascent myotubes with their parent fibers prevents their progressive differentiation during the initial period

Fig. 5 Block of terminal differentiation and degeneration of daughter muscle fibers located beneath the basal laminae of parent muscle fibers. a, b Double indirect immunofluorescent staining for embryonic myosin (a) and laminin (b) in the same field shows the location of a newly formed fiber (asterisk) in 2-month denervated muscle. c Several daughter muscle fibers (arrowheads) located beneath the basal lamina of a parent muscle fiber in 2-month denervated muscle. Short arrows show the location of the basal lamina. d High-resolution view of the area marked with asterisk in c. Note the presence of well-preserved myofibrils in the parent muscle fiber $(p f)$ and poorly developed myofilaments in the daughter fibers. Small arrows show the common basal lamina surrounding both the parent and daughter muscle fibers. Note the presence of the new forming basal lamina on the surface of the parent muscle fiber (arrowheads) and the remnants of degenerated muscle fiber (two asterisks). e A degenerating daughter muscle fiber located beneath the basal lamina (arrowheads) of the parent muscle fiber $(p f)$. The presence of the areas free of organelles (four asterisks) containing a few degenerating mitochondria, vacuoles, and no myofibrils suggests the ongoing degenerative process. Four short arrows show the location of another poorly differentiated muscle fiber located beneath the basal lamina. f A parent fiber with two daughter muscle fibers (arrowheads) under a common basal lamina in 10-month denervated extensor digitorum longus muscle. Short arrows show the remnants of degenerated muscle fibers of small diameter; the long arrow shows an empty basal lamina.(a, b $\times 560, \mathbf{c} \times 5,200, \mathbf{d} \times 11,500, \mathbf{e} \times 16,000, \mathbf{f} \times 8,900)$ 

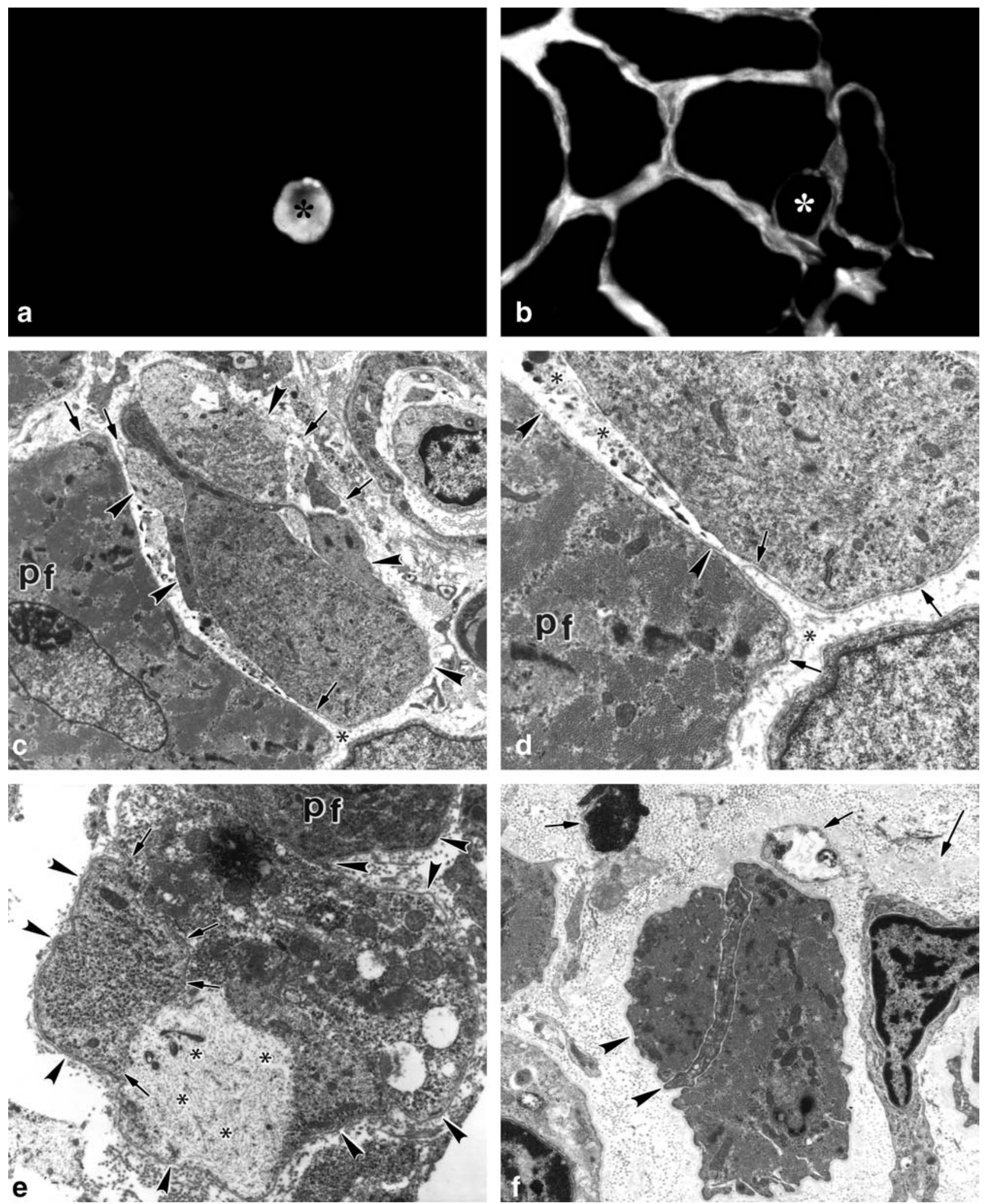

of atrophy. Unlike the first few months of denervation, at late stages of postdenervation atrophy (12-18-month denervated muscle), some of the daughter myotubes had well-developed elements of an organized myofibrillar system. At the same time, even at the advanced stages of postdenervation atrophy, muscle tissue contained degenerating small fibers and empty profiles of basal laminae (Fig. 5f). 
Fig. 6 Formation of multiple newly formed myotubes within the basal lamina in 2-month denervated tibialis anterior muscle. Double immunofluorescent labeling for embryonic myosin (a) and laminin (b) in the same field. Arrows in a show the location of myotubes immunopositive for embryonic myosin, and arrows in $\mathbf{b}$ show the location of the basal lamina profile containing these myotubes. $(\times 97)$
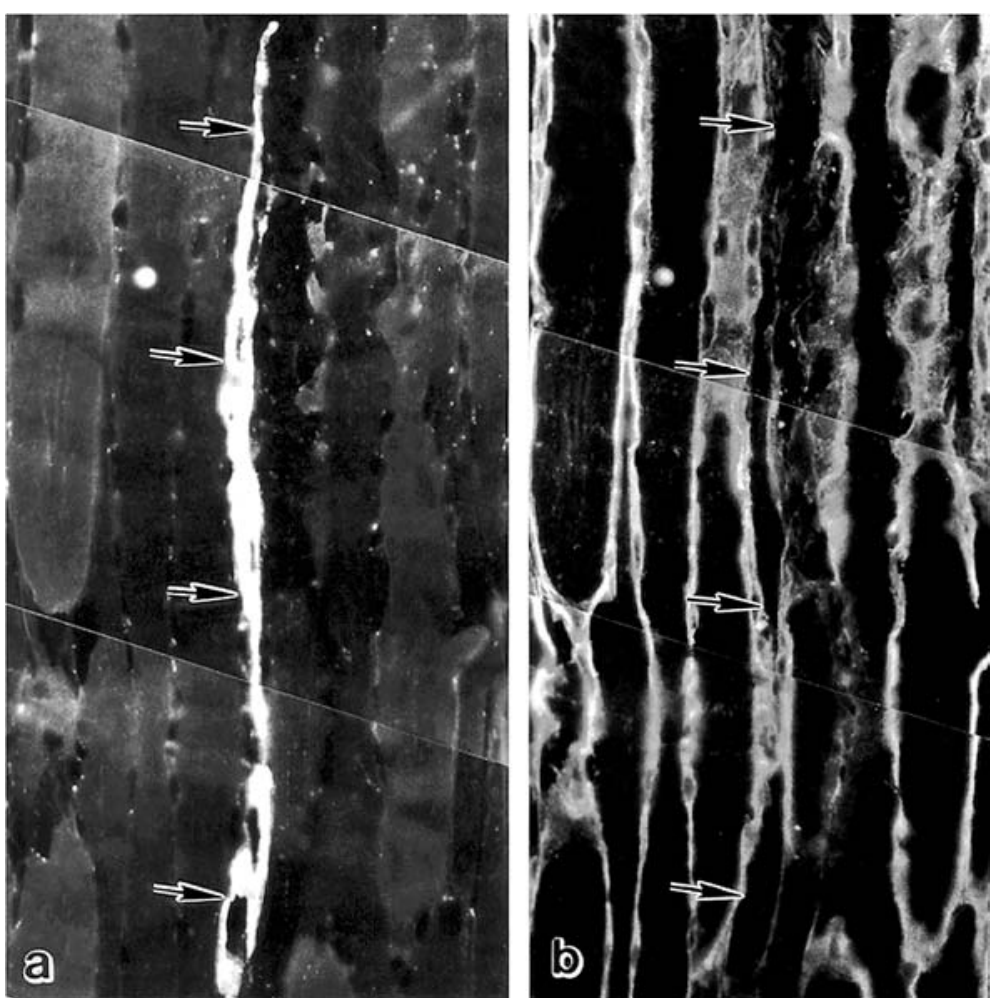

Another manifestation of abortive myogenesis is the formation of clusters of small branching myotubes immunopositive for embryonic myosin and located within the empty spaces of the basal laminae of degenerating muscle fibers (Fig. 6). The fine structure of these cells was very similar to that presented in Fig. $5 \mathrm{c}-\mathrm{e}$. Many of these cells lacked mature sarcomeres, and some of them contained only disassembled myofilaments and poorly differentiated nascent myofibrils similar to the structures shown in Fig. 3b. It remains unclear why activated myoblasts in such situations do not fuse into large fibers. One of the possible explanations is that the remnants of degenerated cells within the sublaminal spaces may create a mechanical obstacle preventing fusion of satellite cells and normal regeneration of muscle fibers. We reported earlier the presence of dead cells and discussed the ultrastructural characteristics of cell degeneration and death in this system (Borisov and Carlson 2000; Borisov et al. 2001) and for this reason do not illustrate this process in the present paper.

It is important to note that muscle fibers retaining a high level of differentiation and little atrophic changes were present both at early and advanced stages of postdenervation atrophy (Figs. 2, 5c, d). These cells typically possessed peripherally located nuclei and a well-preserved contractile system that was aligned in register at the level of Z-bands and occupied the whole volume of the sarcoplasm (Fig. 2). They were surrounded by a single basal lamina, which indicates that they may represent a well-preserved subpopulation of cells resistant to atrophy rather than the new terminally differentiated muscle fibers formed during the compen- satory regeneration. A regenerative process in denervated muscle usually occurs within the basal lamina of degenerated cells and leads to the formation of new muscle fibers surrounded by a double basal lamina (for discussion see Borisov et al. 2001). Relatively large muscle cells with centrally located nuclei and clearly visible myofibrils were also present in long-term denervated tissue (Figs. 1, 2). The level of organization of the contractile system in the fibers with central nuclei was considerably lower than that in the fibers with peripheral nuclei (Fig. 2).

To better understand the cellular mechanism of abortive myogenesis in denervated muscle, we studied the structural characteristics of myoblasts with special reference to the process of their fusion. We found that activation of satellite cells occurred during the first 2 3 weeks following denervation. Structural manifestations of this process included the development of abundant rough endoplasmic reticulum and an increase in cytoplasmic volume of satellite cells (Figs. 7a, b, 9b). Some satellite cells were able to fuse directly with differentiated muscle fibers, and the areas of cytoplasmic fusion can be clearly seen in electron microscopic photographs (Fig. 7b). Despite extensive searches, we did not find any mitotically dividing satellite cells located in their typical position beneath the basal lamina. At the same time, we observed mitotic cells in the interstitial space (Fig. 7c, d). Although most of these cells had a typically fibroblastic morphology, ultrastructurally some of them closely resembled activated satellite cells. This resemblance included well-developed rough endoplasmic reticulum, the presence of fine granulation and 
Fig. 7 Activation of satellite cells in denervated muscle. a, b The presence of well-developed rough cytoplasmic reticulum in the cytoplasm of activated satellite cells. Arrows show the location of satellite cells beneath the basal lamina. Note the large volume of cytoplasm in the cell shown in $\mathbf{b}$, its fusion with a muscle fiber, and the absence of myofibrils in the area of fusion marked with an asterisk. The arrowhead indicates the gap between the myoblast and the muscle fiber near the area of fusion. c, $\mathbf{d}$ Two sections of a mitotic cell located in the endomysial space. This cell has the cytoplasmic ultrastructure very similar to that of satellite cells (compare with the cell located beneath the basal lamina in panels a and $\mathbf{b}$ ). d Higher magnification view of the cell shown in $\mathbf{c}$ and located between two muscle fibers. (a, b $\times 11,000, \mathbf{c} \times 6,730, \mathbf{d} \times 6,610)$
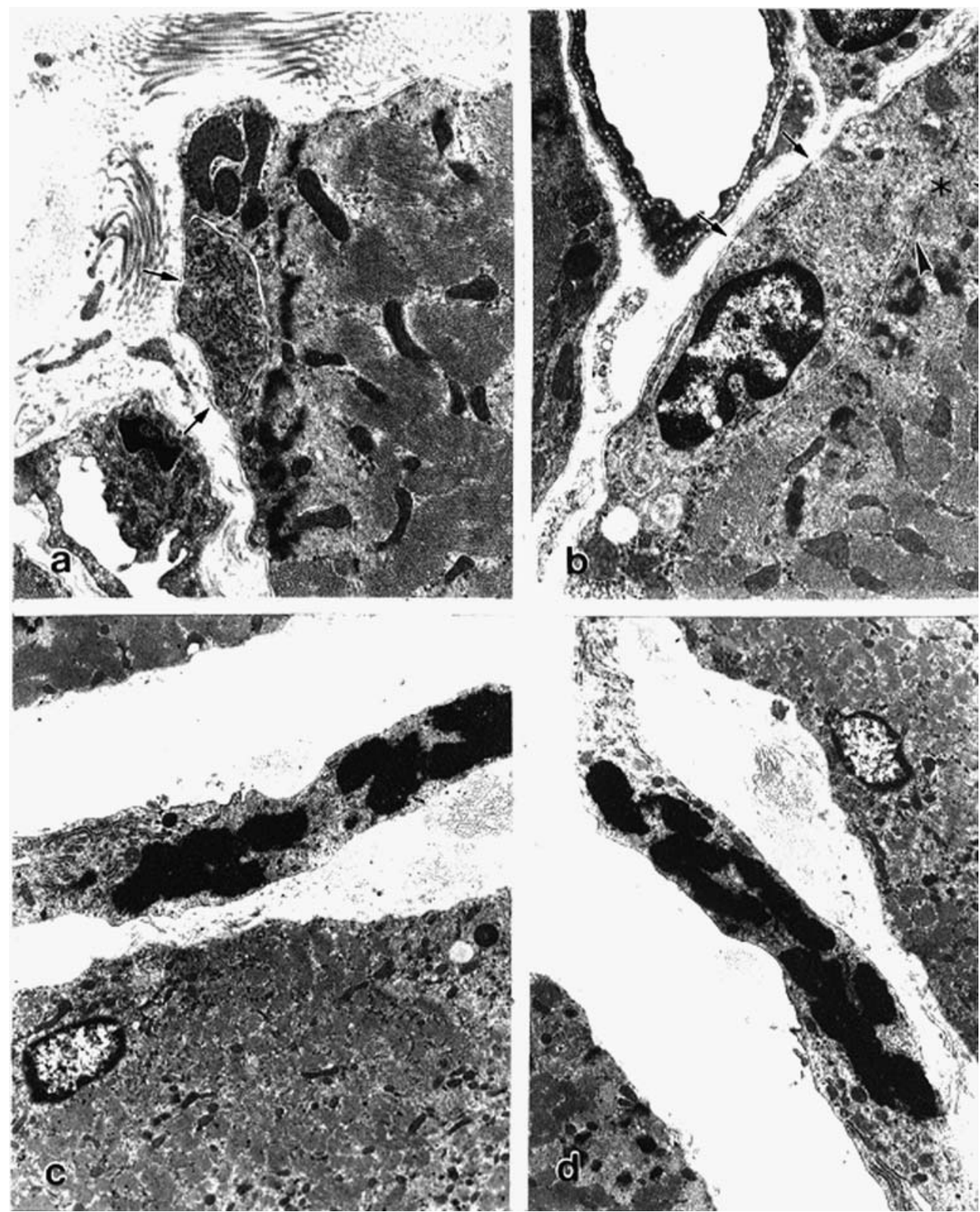

microfilaments in the cytoplasm, and a small number of rounded mitochondria, which is typical of activated satellite cells in their normal sublaminal location (compare Fig. $7 \mathrm{a}-\mathrm{d}$ ). This finding was supported by the results of immunocytochemical labeling of activated satellite cells using an antibody to neural cell adhesion molecule (NCAM; Fig. 8a-c), which confirmed that some of these cells were indeed activated myoblasts localized between muscle fibers in the endomysial space. It has been shown earlier that activated satellite cells intensely express NCAM, and this protein is considered one of their markers (Irintchev et al. 1994; Dedkov et al. 2003).

Thus, we found that formation of small muscle fibers outside the basal lamina can occur in two different ways: (1) by formation of daughter muscle fibers beneath the basal laminae of their parent fibers with subsequent spatial separation, and (2) by fusion of migrating satellite cells in the interstitial space between muscle fibers. In the first case, the process of sequestration of daughter fibers occurs as a result of development of a basal lamina between the parent and daughter muscle fibers, as shown in Fig. 5a-d. In the second case, activated myogenic cells fuse to one another outside the basal laminae of parent muscle fibers (Fig. 8a-c) and form their own basal lamina. Confocal microscopy also demonstrated that some cytoplasmic protrusions of subliminally located satellite cells were located very closely to the outer surface of the basal lamina or even outside its boundaries (Fig. 9a), a finding further supported by our ultrastructural study. Electron microscopy of denervated muscle provided additional evidence of the capacity of myogenic satellite cells for spatial separation from 

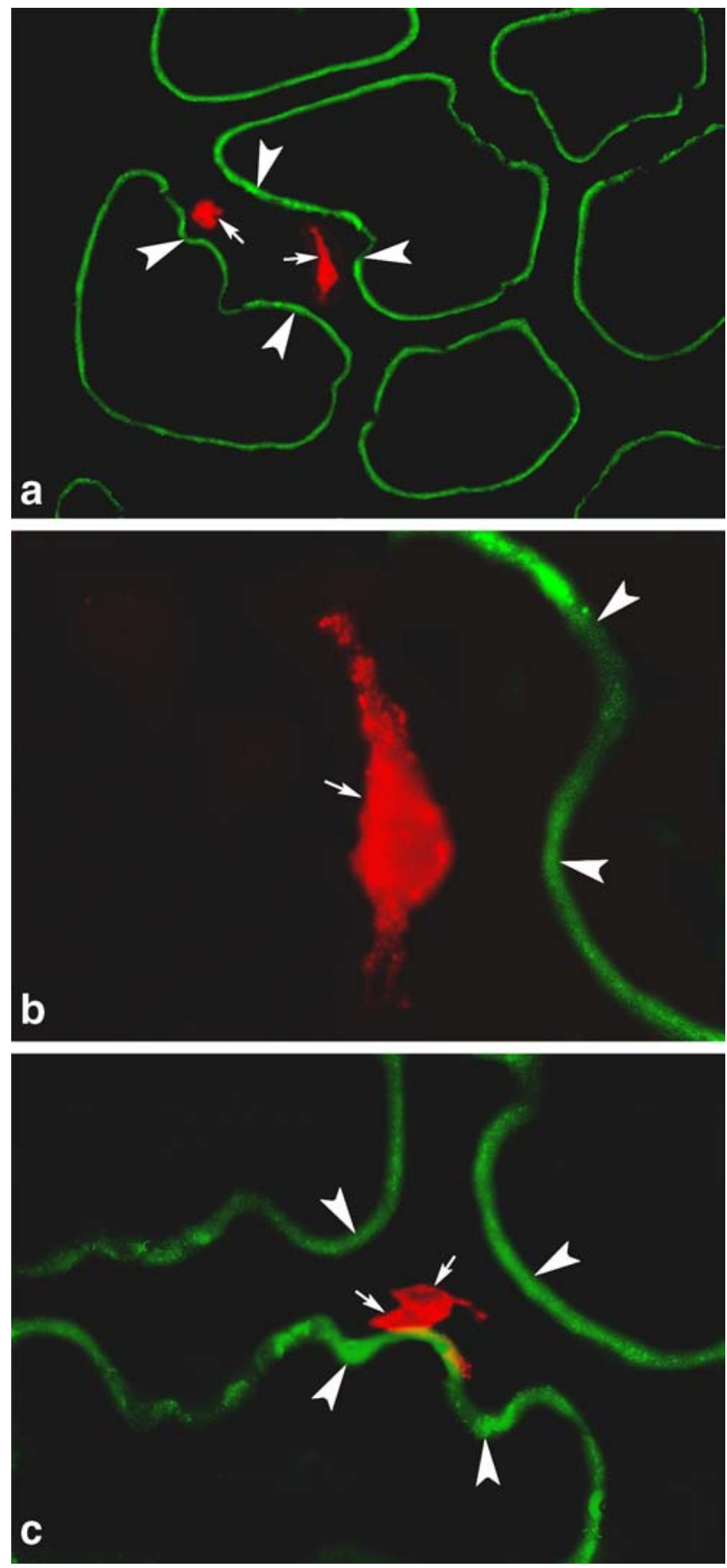

Fig. 8 Migration and fusion of satellite cells in the interstitial space of denervated muscle. a Double immunolabeling for neural cell adhesion molecule (NCAM; red fluorescence) and laminin (green fluorescence) shows the location of activated satellite cells (arrows) in the endomysial space between muscle fibers. Arrowheads show the location of the basal laminae of muscle fibers. b Higher magnification view of a cell shown in a. c Close topographical apposition suggests the fusion of two cells immunopositive for NCAM (arrows) in the interstitial space between muscle fibers. Arrowheads in $\mathbf{b}$ and $\mathbf{c}$ show the discontinuous basal laminae with openings that may allow the satellite cells to migrate. $(\mathbf{a} \times 258$, b $\times 1,250, \mathbf{c} \times 530)$ muscle fibers and their migration through the interstitial space. Some myoblasts extended cytoplasmic processes through the openings in the basal lamina into the endomysial space (Fig. 9b), which indicates that the migration can occur through the openings in the basal lamina. We also found satellite cells at more advanced stages of this process with only point contacts with muscle fibers (Fig. 9c). Interestingly, in some cases at advanced stages of detachment of satellite cells from their normal positions, the basal lamina of the parent muscle fiber frequently remained intact (Fig. 10a, b). This suggests that the process of detachment may precede the separation of myoblasts and local dissolution of the basal lamina. The migration of satellite cells at late stages following denervation occurs in the direction of capillaries and small blood vessels (Fig. 10a, b), which indicates that the process of differentiation of new muscle fibers may require oxygenation. At the same time, the interpretation of the images presented above does not exclude the possibility of migration of satellite cells toward the muscle fibers and formation of the basal lamina following the formation of intercellular contacts.

\section{Discussion}

The results of this study help in understanding why denervated muscle, despite the presence of a large population of satellite cells, undergoes atrophy and fails to restore its structural and functional capacity through the regenerative process. Very little is known regarding the properties of satellite cells in long-term denervated muscles. The available data mainly concern muscles denervated for less than 3-6 weeks. Paradoxically, the number of myogenic satellite cells significantly increases during the first several weeks following denervation (for discussion, see Lu et al. 1997 and Viguie et al. 1997). Activation of DNA synthesis in satellite cells and an increase in their number at early stages following denervation have been reported in mammalian denervated muscles by several groups (for discussion, see Gosselin et al. 1994, Murray and Robbins 1982, Ontell 1974, Schultz 1978 and Snow 1983). Earlier we presented evidence of activation of satellite cells following longterm denervation that includes reexpression of myogenic regulatory factors MyoD and myogenin (Borisov and Carlson 1997) and the presence of centrioles in some satellite cells that we illustrated earlier (Dedkov et al. 2001). In our previous studies, we found that in the rat extensor digitorum longus muscle, the percentage of satellite cells rose from $2.8 \%$ control value to $9.1 \%$ at 2 months of denervation and then progressively decreased to $1.1 \%$ at 18 months (Viguie et al. 1997) and $0.5 \%$ at 25 months of denervation (Dedkov et al. 2001). Thus, by 25 months of denervation, the number of satellite cells decreased more than five times from the values observed in control innervated muscle. Our results indicate that after 5-7 months of denervation, the loss of satellite cells during myogenesis leads to a 

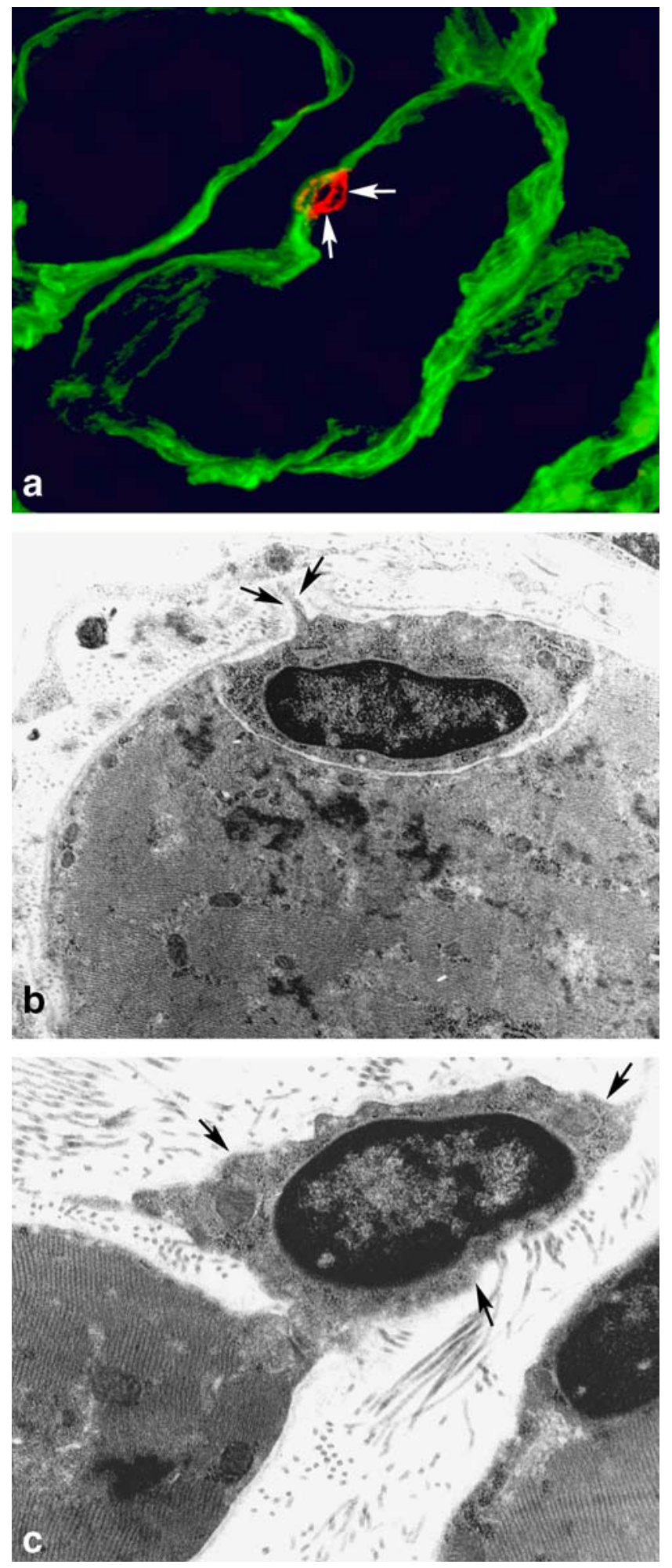

Fig. 9 Migration of satellite cell through the basal lamina. a Confocal image of a satellite cell in its typical position immunolabeled for neural cell adhesion molecule (NCAM; red fluorescence) and located beneath the basal lamina immunolabeled for laminin (green fluorescence). Note the presence of NCAM immunopositivity localized very close to the outer surface of the basal lamina. b Electron micrograph of a cell in a typical position for a satellite, extending a cytoplasmic process through the opening in the basal lamina of a muscle fiber (arrows). c Electron micrograph of a mononucleated cell (arrows) locally contacting a muscle fiber. Note the absence of the basal lamina on the cell's frontal edge. $(\mathbf{a} \times 560, \mathbf{b}$ $\times 6,300, \mathbf{c} \times 11,630)$
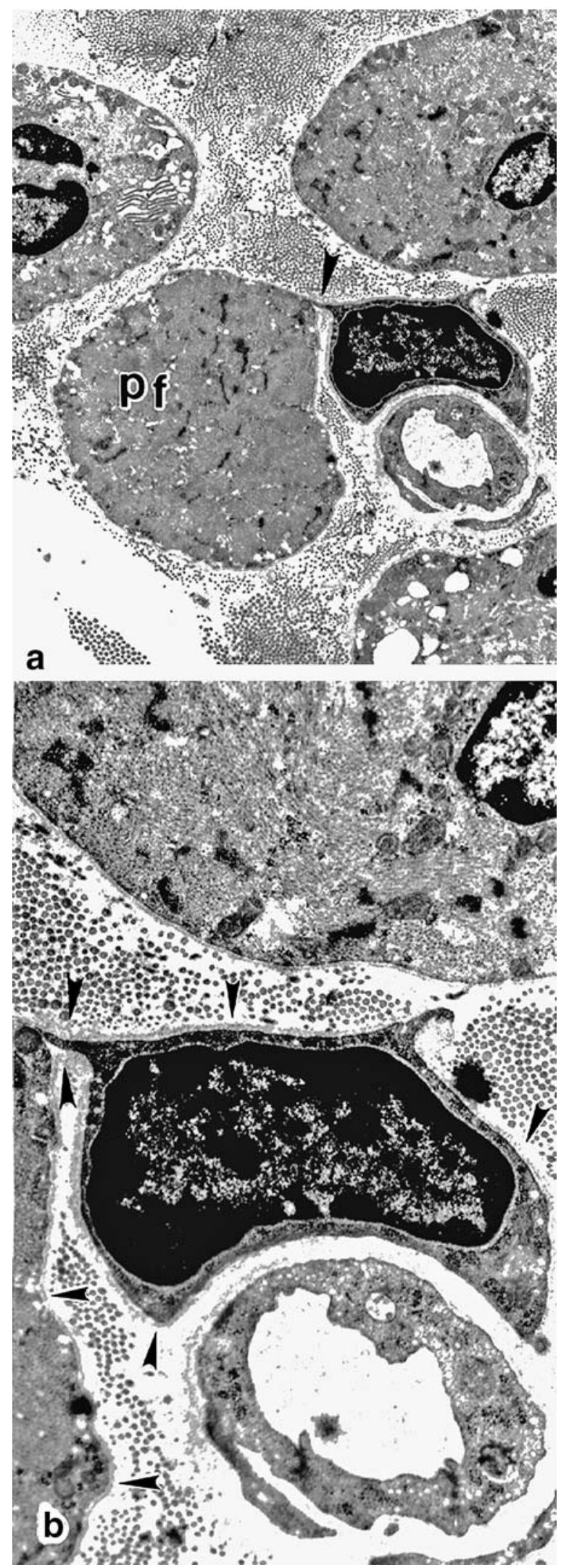

Fig. 10 The process of myoblast migration from or to muscle fibers. a Arrowhead shows a small bridge connecting a mononucleated myoblast and an atrophic muscle cell labeled as a parent fiber $(p f)$. b Higher magnification of the area connecting two cells shown in a. Arrowheads indicate the intact common basal lamina covering both the myoblast and a muscle cell. Also note a close apposition of the satellite cell and a capillary. $(\mathbf{a} \times 3,300, \mathbf{b} \times 7,820)$ 
progressive decrease in their number. This suggests that denervation results in the activation of an uncontrolled myogenic response and ultimate exhaustion of the pool of satellite cells.

The presence in our material of the differentiated fibers surrounded by a double basal lamina indicates that individual regenerated muscle cells can attain advanced levels of differentiation (Borisov et al. 2001). Having found muscle cells with doubled basal laminae in rat soleus muscle, Schmalbruch et al. (1991) concluded that the original fibers are replaced by a population of regenerated fibers during the postdenervation period. Our observations of the extensor digitorum longus and tibialis anterior muscles indicate that the number of fibers with double basal laminae was relatively low in these two fast muscles compared with that observed in the soleus. This appears to be explained by the difference in responses of fast and slow muscles to denervation. The migratory ability of myoblasts has been demonstrated during skeletal muscle development (Hughes and Blau 1990) and regeneration (Watt et al. 1987; Rando et al. 1995). To this end, migration of satellite cells in denervated muscle recapitulates their behavior during the developmental myogenesis.

We found four types of involvement of satellite cells in a myogenic response following denervation: (1) formation of new myotubes on the surface of live terminally differentiated muscle fibers, (2) regeneration of new muscle fibers within the basal laminae of degenerated fibers, (3) formation of new muscle fibers by migrating myoblasts outside the basal lamina, and (4) fusion of satellite cells with atrophying muscle fibers. The primary factor blocking the terminal differentiation of muscle fibers under conditions of denervation remains unclear. Recently, we have shown that activation of myogenesis in denervated muscle does not require significant atrophy, degeneration, and death of muscle fibers (Borisov et al. 2001). Electrical or trophic effects of the motor nerve and a lack of functional activity may significantly affect the differentiative properties of nascent muscle cells. At the same time, many questions still remain unanswered concerning the regulatory interactions of satellite cells and muscle fibers and the mechanisms initiating activation of myogenic cells during the regenerative process (Bischoff 1990, 1994; Schultz and McCormic 1994). Other possible factors may involve changes in the properties of the basal laminae or local modulations of expressions of mitogenic molecules. During the normal regenerative process in skeletal muscle, the basal lamina serves as a scaffold for orderly cell replacement (Vracko et al. 1972). Some modifications of the basal lamina have been observed during muscle fiber degeneration and regeneration (Gulati et al. 1983). No information is available as yet about the changes of expression of basal lamina proteins following denervation. It is apparent that several of these proteins, such as different isotypes of laminin, may play an important role in regulating proliferation and differentiation of myogenic cells. Localization of immunoreac- tivity for basic fibroblast growth factor near satellite cells and regenerating muscle fibers in myodystrophic mdx mice demonstrated by Anderson et al. (1991) indicate the possibility of autocrine regulation of muscle growth. In addition, the local gradients of mitogen concentrations may explain the topographical heterogeneity of myogenic response in denervated muscle and the presence of intermingled myogenically active and quiescent zones within denervated muscle tissue. Another important factor that may block normal regeneration and terminal differentiation of muscle fibers is the impairment of vascularization in denervated muscle. We found that 7-9 months following denervation, 85-90\% of capillaries completely degenerated, and significant degenerative changes occurred in arterioles and venules (Borisov et al. 2000; Dedkov et al. 2002). The development of avascular zones in long-term denervated muscle appears to be associated with a foci of hypoxia that may prevent the regenerative process.

Progressive interstitial fibrosis and accumulation of dense deposits of collagen among muscle fibers during the postdenervation period in vivo can create a mechanical obstacle for migration, fusion, and normal terminal differentiation of activated myogenic cells. Similarly, the presence of incompletely resorbed fragments of degenerated muscle fibers within the sublaminal space appears to be another factor preventing normal fusion and differentiation of myoblasts. This explains why clusters of small muscle fibers or branching fibers rather than normally regenerating cells are formed in the empty basal laminae of degenerated muscle fibers. Taken together, all the factors discussed above can contribute to the progressive irreversible decline of the regenerative capacity and the ability for functional restoration typical of long-term denervated skeletal muscle.

After 5-7 months of denervation, the loss of satellite cells during the course of abortive myogenesis results in a decrease in their number and progressive spatial separation. Exhaustion of the satellite cell pool in long-term denervated muscle may also impair normal differentiation of muscle fibers. Another possible explanation of the abortive character of myogenesis in long-term denervation muscle is the lack of community effect; i.e., an insufficient number of precursor cells required for normal differentiation. The community effect has been described during differentiation of cells belonging to different histogenetic lineages, including myoblasts (Cossu et al. 1995; Carnac and Gurdon 1997; Standley et al. 2001). It is interesting to mention that the formation of morphologically abnormal branching muscle fibers has been described during regeneration of aging skeletal muscle (Blaivas and Carlson 1991). In young and old normal muscles, only a very low percentage of muscle fibers are branched (Snow and Chortkoff 1987). Aging is accompanied by impairment of denervation and activation of degenerative changes in motor neurons (for discussion see Larsson and Ansved 1995). These data suggest that the structural abnormalities of regenerative myogenesis observed in denervated and aging 
muscle are explained by the same factors. Thus, the results of this study show the fate of satellite cells in denervated muscle and provide an explanation of why muscle tissue irreversibly loses the ability for regeneration and restoration following several months of denervation.

Acknowledgment This work was supported by NIH grant P01AG10821.

\section{References}

Anderson JE, Liu L, Kardami E (1991) Distinctive patterns of basic fibroblast growth factor (bFGF) distribution in degenerating and regenerating areas of dystrophic $(\mathrm{mdx})$ striated muscles. Dev Biol 147:96-109

Bischoff R (1990) Interaction between satellite cells and skeletal muscle fibers. Development 109:943-952

Bischoff R (1994) The satellite cell and muscle regeneration. In: Engel AG, Franzini-Armstrong C (eds) Myology basic and clinical. McGraw-Hill, New York, pp 97-118

Blaivas M, Carlson BM (1991) Muscle fiber branching - difference between grafts in old and young rats. Mech Ageing Dev 60:4353

Borisov AB, Carlson BM (1997) Abortive myogenesis in denervated skeletal muscle. FASEB J 11:A420 (abstract)

Borisov AB, Carlson BM (2000) Cell death in denervated skeletal muscle is distinct from classical apoptosis. Anat Rec 258:305318

Borisov AB, Huang S-K, Carlson BM (2000) Remodeling of the vascular bed and progressive loss of capillaries in denervated skeletal muscle. Anat Rec 258:292-304

Borisov AB, Dedkov EI, Carlson BM (2001) Interrelations of myogenic response, progressive atrophy of muscle fibers, and cell death in denervated skeletal muscle. Anat Rec 264:203-218

Carlson BM, Faulkner JA (1988) Reinnervation of long-term denervated muscle freely grafted into an innervated limb. Exp Neurol 102:50-56

Carnac G, Gurdon JB (1997) The community effect in Xenopus myogenesis is promoted by dorsalizing factors. Int J Dev Biol 41:521-524

Cossu G, Kelly R, DiDonna S, Vivarelli E, Buckingham M (1995) Myoblast differentiation during mammalian somitogenesis is dependent upon a community effect. Proc Nat Acad Sci USA 92:2254-2258

Dedkov EI, Kostrominova TY, Borisov AB, Carlson BM (2001) Reparative myogenesis in long-term denervated skeletal muscles of adult rats results in reduction of satellite cell population. Anat Rec 263:139-154

Dedkov EI, Kostrominova TY, Borisov AB, Carlson BM (2002) Resistance vessel remodeling and reparative angiogenesis in the microcirculatory bed of long-term denervated skeletal muscle. Microvasc Res 63:96-114

Dedkov EI, Borisov AB, Wernig A, Carlson BM (2003) Aging of skeletal muscle does not affect the response of satellite cells to denervation. J Histochem Cytochem 51:853-863
Gosselin LE, Brice G, Carlson B, Prakash YS, Sieck G (1994) Changes in satellite cell mitotic activity during acute period of unilateral diaphragm denervation. Am J Physiol 77:1128-1134

Gulati AK, Reddi AH, Zalewski AA (1983) Changes in the basement membrane zone components during skeletal muscle fiber degeneration and regeneration. J Cell Biol 97:957-962

Hughes SM, Blau HM (1990) Migration of myoblasts across basal lamina during skeletal muscle development. Nature 345:350 353

Irintchev A, Zeschnigk M, Starzinski-Powitz A, Wernig A (1994) Expression pattern of M-cadherin in normal, denervated, and regenerating mouse muscles. Dev Dyn 199:326-337

Larsson L, Ansved T (1995) Effects of aging on the motor unit. Prog Neurobiol 45:397-458

Lu D-X, Huang S-K, Carlson BM (1997) Electron microscopy of long-term denervated rat skeletal muscle. Anat Rec 248:355365

Murray MA, Robbins N (1982) Cell proliferation in denervated muscle: identity and origin of dividing cells. Neuroscience 7:1823-1833

Ontell M (1974) Muscle satellite cells: a validated technique for light microscopic identification and a quantitative study of changes in their population following denervation. Anat Rec 178:211-228

Rando TA, Pavlath GK, Blau HM (1995) The fate of myoblasts following transplantation into mature muscle. Exp Cell Res 220:383-389

Reynolds ES (1963) The use of lead citrate at high pH as an electron-opaque stain in electron microscopy. J Cell Biol 17:208213

Schmalbruch H, Al-Amood WS, Lewis DM (1991) Morphology of long-term denervated rat soleus muscle and the effect of chronic electrical stimulation. J Physiol 4441:233-241

Schmalbruch H, Lewis DM (1994) A comparison of the morphology of denervated with aneurally regenerated soleus muscle of the rat. J Mus Res Cell Motil 15:256-266

Schultz E (1978) Changes in the satellite cells of growing muscle following denervation. Anat Rec 190:299-312

Schultz E, McCormick KM (1994) Skeletal muscle satellite cells. Rev Physiol Biochem Pharmacol 123:214-257

Snow MH (1983) A quantitative ultrastructural analysis of satellite cells in denervated fast and slow skeletal muscles of the mouse. Anat Rec 207:593-604

Snow MH, Chortkoff BS (1987) Frequency of bifurcated muscle fibers in hypertrophic rat soleus muscle. Muscle Nerve 10:312317

Standley HJ, Zorn AM, Gurdon JB (2001) eFGF and its mode of action in the community effect during Xenopus myogenesis. Development 128:1347-1357

Viguie CA, Lu D-X, Huang S-K, Rengen H, Carlson BM (1997) Quantitative study of the effects of long-term denervation on the extensor digitorum longus muscle of the rat. Anat Rec 248:346-354

Vracko R, Benditt EP (1972) Basal lamina: the scaffold for orderly cell replacement. Observation on regeneration of injured skeletal muscle fibers and capillaries. J Cell Biol 55:406-419

Watt DJ, Morgan JE, Clifford MA, Partridge TA (1987) The movement of muscle precursor cells between adjacent regenerating muscles in the mouse. Anat Embryol (Berl) 175:527-536 